\title{
Structural studies on hydrating cement pastes
}

\author{
F. EICHHORN, F. HÄUSSLER ${ }^{*},(1)$ and H. BAUMBACH*
}

Research Center Rossendorf Inc., Institute of Ion Beam Physics and Materials Research, P.O. Box 19, 8051 Dresden, Germany

* Institute for Nondestructive Testing Saarbrücken (FhG), University, Building 37, 6600 Saarbrücken, Germany

\begin{abstract}
The development of the mesoscopic material structure during the hydration of ordinary Portland cement paste is studied by small-angle neutron scattering. Results on the time dependence of radii of gyration (Guinier region) and exponents (Porod region) are given and discussed.
\end{abstract}

\section{Introduction}

Building materials like hardened cement are heterogeneous. Their useful properties (as strength, porosity, thermal insulation permeability for gases and liquids) are determined by the structure within and between their components. The development of the material structure during the hydration process depends on the starting values (composition of the mixture) and on the external physical parameters and technological conditions of the process (e. g. temperature, humidity, vibration).

To observe the mesoscopic structures in an averaged way the method of small-angle neutron scattering (SANS) was used with its possibility for contrast variation between different phases of the hydrating material. Hydration with $\mathrm{H}_{2} \mathrm{O}, \mathrm{D}_{2} \mathrm{O}$ or a mixture of them, respectively, gives various values of the scattering length density.

In the last years several publications about SANS measurements on hardening cement pastes, ceramics, and other porous materials were published [1], [2], [3], [6].

Portland cement paste consists of many crystalline and non-crystalline phases in different sizes. In this paper the structure development in hydrating cement paste and methodological aspects of the experiment and its interpretation are considered.

The interaction of the neutron beam with the microstructural objects are characterized by specific properties of the cement material discussed below

\section{SANS Experiments and Material}

Here results of an ordinary Portland cement (type 1/40 A) are presented which are obtained by the MURN device at the IBR-2 pulsed reactor of the Joint Institute for Nuclear Research Dubna/Russia [7]. Using the time-of-flight method SANS curves are measured in the scat-

(1) Present address: Joint Institute for Nuclear Research, Frank Laboratory of Neutron Physics, 141980 Dubna/Moscow Region, Russia 
tering vector range between $Q_{\min }=0.09 \mathrm{~nm}^{-1}$ and $Q_{\max }=1.5 \mathrm{~nm}^{-1}$ with $\delta Q=0.014 \mathrm{~nm}^{-1}$. According to the sampling theorem [4] reliable results from this measuring conditions can be obtained for spherical particles with radii between 1 and $100 \mathrm{~nm}$.

For the the first part of the investigations the samples are prepared from ordinary Portland cement $(O P C)$ and heavy water $\left(\mathrm{D}_{2} \mathrm{O}\right)$ with a water-cement ratio of 0.38 . For these preparation conditions the most abundant hydrated cement phases $C_{3} S_{2} \cdot 2.5 \mathrm{w}$ (content 45 per cent), $\mathrm{Ca}(\mathrm{OH})_{2}$ (22 per cent), and $\mathrm{C}_{3} \mathrm{~A} \cdot \mathrm{CaSO}_{4} \cdot 12 \mathrm{~W}$ (17 per cent) give the maximum contrast with the pore system $\left(C=\mathrm{CaO}, S=\mathrm{SiO}_{2}, A=\mathrm{Al}_{2} \mathrm{O}_{3}\right.$, and $w=$ water) [5]. The thicknesses of the specimens used were $0.5 \mathrm{~mm}, 1.05 \mathrm{~mm}$, and $1.7 \mathrm{~mm}$.

By means of a vanadium scatterer placed into the direct neutron beam the macroscopic cross section $d \Sigma(Q) / d \Omega$ is measurable, it contains all scattering information about the sample.

\section{Study of Hardening Cement Pastes}

Portland cement paste contains many crystalline and non-crystalline phases in various ranges of sizes. It is possible to divide the hydrating cement grain into several shells for the modeling. The shells represent the inner and outer hydration products. The crystalline phases (e.g. Portlandite, Calcite) are embedded in the amorphous phases of hydration products. The nucleus of the hydrating grain consists of unreacted Portland cement.

From the point of view of the interaction between the thermal neutrons and the microstructural objects the principal properties of the samples of hardened cement paste under investigation by SANS are:

1. Heterogeneity: The sample typically contains a full variety of phases (unreacted cement grain, various hydration products, electrolytic solutions) which appear as a result of the hydration process between the cement clinker and the water. A selection of the most important scattering objects is necessary for an effective interpretation of the measured SANS data.

2. Scattering objects of a size range of some micrometers: Both dry cement and hardened cement paste contain scattering objects which are in a size range larger than some $\mu \mathrm{m}$. Therefore the conditions for application of the first BORN approximation has to be considered.

3. Dense packed system: It is thought that even a single clinker grain consists of different phases and represents a dense packed system of heterogeneous objects. The neutrons travelling through the sample feel a fast change of scattering objects i.e. scattering length densities. A priori the interparticle structure factor should not be neglected because of the very dense packing in the system.

4. Sample thickness: To neglect the effects of sample surface roughness and emphasize the sample volume effects, the sample thickness must be at least of $1 \mathrm{~mm}$.

5. Evolution of the hydrating phases: A continuous evolution of the hydrating phases is observable. Only special sample preparations may stop this evolution. The sensitivity of dry cement powder and hardened cement paste to various outer and inner influences needs the use of difficult preparation procedures (e.g. a chamber for controlling the humidity, temperature etc.). 


\section{Results}

During a longer period the structure development of hydrating cement paste was investigated. Figures 1 and 2 show the measured macroscopic scattering cross sections $d \Sigma / d \Omega$. Cement hydration is an exothermic process. Therefore, the structure parameters (e.g. the radius of gyration $R_{\text {gsat }}$ for $t \rightarrow \infty$ given in Table 1) differ for samples of different thickness. The dependence on the time $t$ of such structure parameters is given by exponential laws

$$
\begin{aligned}
& R_{g}=R_{g s a t} \cdot\left(1-\exp \left(-t / T_{g}\right)\right) \\
& d \Sigma / d \Omega_{Q=0}=d \Sigma / d \Omega_{0 s a t} \cdot\left(1-\exp \left(-t / T_{0}\right)\right) .
\end{aligned}
$$

Table 1:

Radius of gyration $R_{g s a t}$ and the scattering cross section $d \Sigma / d \Omega$ at $Q=0$ for the samples used and their time parameters

\begin{tabular}{|c|cccc|}
\hline $\begin{array}{c}\text { Thickness of } \\
\text { the sample } \\
/ \mathrm{mm}\end{array}$ & $R_{\text {gsat }}$ & $T_{g}$ & $d \Sigma / d \Omega_{0 s a t}$ & $T_{0}$ \\
\hline & $/ \mathrm{nm}$ & $/ \mathrm{d}$ & $/ \mathrm{cm}^{-1}$ & $/ \mathrm{d}$ \\
\hline 0.50 & 13.6 & 0.66 & 470 & 4.98 \\
1.05 & 12.6 & 0.58 & 218 & 6.00 \\
1.70 & 11.4 & 0.32 & 172 & 8.89 \\
\hline
\end{tabular}

Assuming a logarithmic normal distribution for the size (geometric radius $\mathrm{R}$ ) of spherical particles (pores or grains of different phases)

$$
N(R)=N_{g} / \sqrt{2 \pi} p R \cdot \exp \left[\ln \left(R / R_{0}\right) / p\right]^{2} / 2
$$

the most probable radius $R_{\circ}$ (centre of the distribution) and the dispersion of that distribution $(p \sim$ FWHM) are given in the Table 2.

Table 2:

Parameters (the most probable radius $R_{0}$ and the FWHM of a logarithmic normal distribution for spherical particles in dependence on the hydration time of Portland cement paste. The thickness of the sample was $0.5 \mathrm{~mm}$.

\begin{tabular}{|c|cc|}
\hline $\begin{array}{c}\text { Hydration time } \\
/ \mathrm{d}\end{array}$ & $\begin{array}{c}R_{o} \\
/ \mathrm{nm}\end{array}$ & $\begin{array}{c}F W H M \\
/ \mathrm{nm}\end{array}$ \\
\hline & & \\
1 & $3.3 \pm 0.1$ & $3.1 \pm 0.1$ \\
60 & $2.8 \pm 0.5$ & $4.3 \pm 0.2$ \\
92 & $3.2 \pm 0.4$ & $4.5 \pm 0.1$ \\
\hline
\end{tabular}

The results show that the mean particle size reaches its final value already in the early stage of hydration whereas the dispersion of the particle size increases during longer periods. Analyzing the scattering cross section at higher values of $Q$ a deviation from the Porod-law (valid for compact particles with sharp boundaries) is observed. This may be caused by 
fractal-like surface or volume structure [3]. To study this problem further investigations are in preparation.
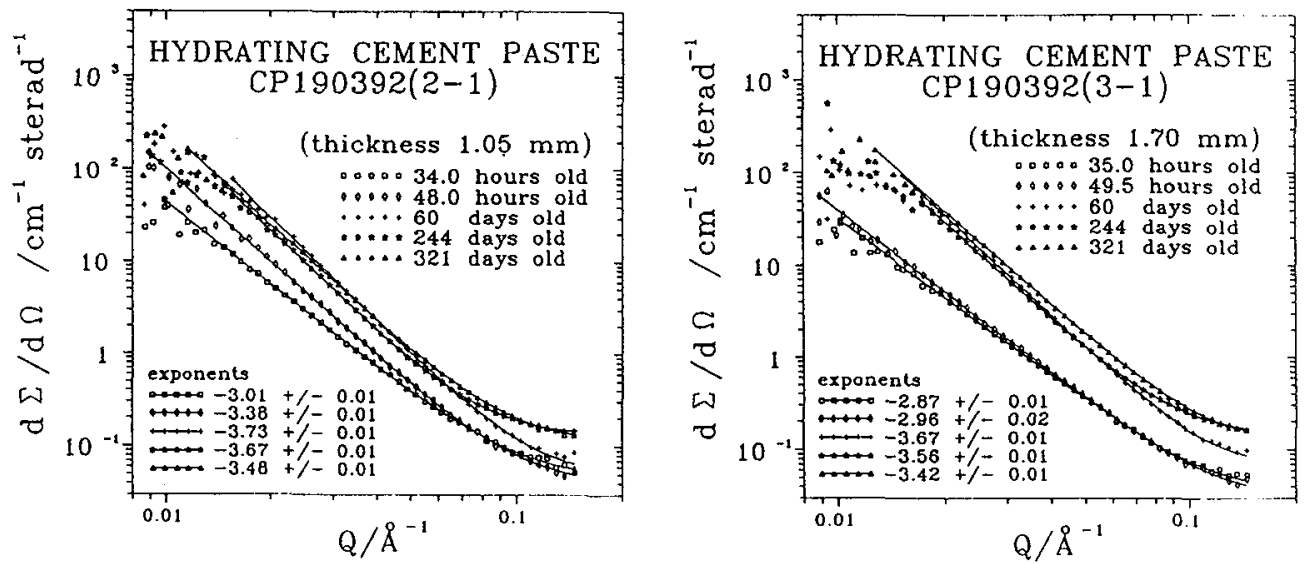

Figures 1 and 2:

Small-angle neutron scattering cross section of a hardening paste of ordinary Portland cement with sample thicknesses of $1.05 \mathrm{~mm}$ and $1.70 \mathrm{~mm}$. The curve parameter is the time after the beginning of the hydration. It can be clearly seen the increase of the linear size of the inhomogeneities during the hydration.

\section{Acknowledgements}

The authors gratefully acknowledge Prof. Yu. M. Ostanevich (Frank Laboratory of Neutron Physics of the JINR Dubna) for his permanent interest, the fruitful discussions and the support of this research. They thank Mrs. A. Hempel (RC Rossendorf) and Mrs. M. Hempel (INT Saarbrücken) for the help in experimental and computing works.

This work was partially supported by the Bundesminister für Forschung und Technologie through Grants No. 03-DU3FHG and No. 03-EI3ROS. The authors are fully responsible for the content of this publication.

\section{References}

[1] A. J. Allen, R. C. Oberthur, D. Pearson, P. Schofield, and C. R. Wilding, Philosophical Magazine B 56 (1987) 263

[2] F. Häussler, F. Eichhorn, S. Röhling, and H. Baumbach, Cement and Concrete Research 20 (1990) 644

[3] A. J. Allen, J. Appl. Cryst. 24 (1991) 62

[4] Th. Gerber, P. W. Schmidt, J. Appl. Cryst. 16 (1983) 581

[5] A. J. Allen, C. G. Windsor, V. S. Rainey, D. Pearson, D. D. Double, and N. McN. Alford, J. Phys. D 15, (1982) 1817

[6] F. Häussler, H. Baumbach, and F.Eichhorn, Proc. VTT Symp., VTT-115 (1990) 102

[7] User Guide "Neutron Experimental Facilities at JINR", LNP-JINR Dubna/Russia 1991, p. 22 